\title{
2021: Computing's Divided Future
}

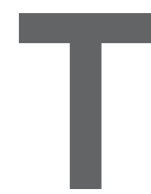

HROUGH THE FOG of headline-grabbing tweets and TikTok ban lawsuits, we can see the tectonic plates of China's and the West's computing ecosystems rapid movement apart. The growing importance of computing as a military and intelligence technology makes this inevitable; compounded by its pervasive presence in society, commerce, and government. At this writing, China continues aggressive actions to eliminate democracy in Hong Kong and military demonstrations to intimidate Taiwan and other nations in Southeast Asia. ${ }^{a}$ China's government has reined in Chinese technology companies such as Ant Financial (Alibaba) and Tencent, overtly signaling its intent for full control. ${ }^{\mathrm{b}}$ These actions also affect foreign multinationals operating in China (for example, Apple and $\mathrm{AirBnB}^{\mathrm{c}}$ ). The action is two-sided with the U.S. government acting to restrict exports based on critical technologies (semiconductors), disrupting visa programs for students and visitors, and increasing reporting requirements for foreign engagements. U.S. government prosecution of highprofile researchers for undisclosed ties and payments has had a chilling effect on collaboration with researchers

a A. Ramzy, T. May, and E. Yu. China targets Hong Kong's lawmakers as it squelches dissent. New York Times (Nov. 11, 2020). China sends warning to Taiwan and U.S. with big show of air power. New York Times (Sept. 18, 2020). R. Zhong. In halting ant's IPO, China sends a warning to business. New York Times (Nov. 6, 2020).

b R. Liao. China finally grants a game license to Tencent. TechCrunch (Jan. 24, 2019).

c S. Liao. Apple officially moves its Chinese iCloud operations and encryption keys to China. The Verge (Feb. 29, 2020). I.C. Campbell. Airbnb's Chinese data policies reportedly cost it an executive. The Verge (Nov. 20, 2020) in China. ${ }^{\mathrm{d}}$ And, the global COVID-19 pandemic has only exacerbated misinformation and conflict.

Recent events have framed starkly these concerns in a scope expanding from hardware technology to software (even algorithms in the case of TikTok) to user-data collection. As we enter a new year, China and the West's fundamental systemic differences and growing geopolitical competition are increasingly open. The new reality is pulling the computing community apart, and yes, spilling over into the academic and research communities. Some analysts project rapid evolution from one computing community to two Internets and then into two business and technology ecosystems, and ultimately "decoupling" into two largely disjoint technology bases. ${ }^{\text {e }}$ The growing schism is far wider, but computing is unavoidably at ground zero. The fissures have grown over time, but their growth has definitely accelerated over the past two years. ${ }^{\mathrm{f}}$ Computing faces a growing divide, and the computing community confronts shifts from Open Collaboration to Coopetition and perhaps to pure Competition.

What are the implications for multinational organizations such as the ACM? For community? And for individuals who seek to study and collaborate internationally? Perhaps open

d N. Ord. TSMC reportedly strikes deal with U.S to supply chips to Huawei but with a caveat. Hot Hardware (Oct. 9, 2002). NPR. Acclaimed Harvard scientist is arrested, accused of lying about ties to China (Jan. 28, 2020).

e A. Hoecker, S. Li, and J. Wang. U.S. and China: The DecouplingAccelerates. Bain \& Co., (Oct. 14, 2020).

f R. Singel and D. Kravets. Only Google could leave China. WIRED (Jan. 15, 2010). A. Chien. Sustaining open collaboration in universities. Commun. ACM (Sept. 2019). A. Chien. Cracks in open collaboration. Commun. ACM (Jan. 2020).

$\mathrm{g}$ E. Harris. Governance of Dual-Use Technologies: Theory and Practice. AAAS, 2016. collaboration is "paradise lost"; realists clearly see the emergence of a bipolar computing world. What should we do?

- Be aware. Just as corporate employees need to adjust for internal and external conversations, researchers working in sensitive areas need to exercise discretion.

- Differentiate carefully. Separating basic research from areas that might be sensitive for national security may avoid chilling restrictions.

- Act responsibly. Egregious abuse will trigger greater oversight and restrictions, and erosion of open scientific collaboration

None of these issues are unique to computing. Geopolitics' impact on scientific collaboration has strong, parallel precedents in physics (nuclear weapons) and biology (biological weapons). Can we do better? Computing's culture of open collaboration is a rich legacy of technology and community. It has a rich web of personal relationships. Can this web help us avoid the harshest outcomes?

Temper distrust, secrecy, and competition by keeping the humanity of your colleagues in mind. As tensions rise, many individuals will face difficult choices torn by comflicting loyalties. As individuals we all want to survive and thrive-personally and professionally. But each of us do so within imperfect systems - perhaps not to our liking and certainly beyond our control.

Let's work to preserve computing's community!

Andrew A. Chien, EDITOR-IN-CHIEF

Andrew A. Chien is the William Eckhardt Distinguished Service Professor in the Department of Computer Science at the University of Chicago, Director of the CERES Center for Unstoppable Computing, and a Senior Scientist at Argonne National Laboratory. 